\title{
A note on transitive union-closed families.
}

\author{
James Aaronson
}

Mathematical Institute

University of Oxford

United Kingdom

james. aaronson.maths@gmail.com

\author{
David Ellis \\ School of Mathematics \\ University of Bristol \\ United Kingdom \\ david.ellis@bristol.ac.uk
}

\author{
Imre Leader \\ Department of Pure Mathematics and Mathematical Statistics \\ University of Cambridge \\ United Kingdom \\ i.leader@dpmms.cam.ac.uk
}

Submitted: Oct 18, 2020; Accepted: Mar 18, 2021; Published: Apr 9, 2021

(C) The authors. Released under the CC BY-ND license (International 4.0).

\begin{abstract}
We show that the Union-Closed Conjecture holds for the union-closed family generated by the cyclic translates of any fixed set.
\end{abstract}

Mathematics Subject Classifications: 05D05

\section{Introduction}

If $X$ is a set, a family $\mathcal{F}$ of subsets of $X$ is said to be union-closed if the union of any two sets in $\mathcal{F}$ is also in $\mathcal{F}$. The celebrated Union-Closed Conjecture (a conjecture of Frankl [2]) states that if $X$ is a finite set and $\mathcal{F}$ is a union-closed family of subsets of $X$ (with $\mathcal{F} \neq\{\varnothing\}$ ), then there exists an element $x \in X$ such that $x$ is contained in at least half of the sets in $\mathcal{F}$. Despite the efforts of many researchers over the last forty-five years, and a recent Polymath project [5] aimed at resolving it, this conjecture remains wide open. It has only been proved under very strong constraints on the ground-set $X$ or the family $\mathcal{F}$; for example, Balla, Bollobás and Eccles [1] proved it in the case where $|\mathcal{F}| \geqslant \frac{2}{3} 2^{|X|}$; more recently, Karpas [4] proved it in the case where $|\mathcal{F}| \geqslant\left(\frac{1}{2}-c\right) 2^{|X|}$ for a small absolute constant $c>0$; and it is also known to hold whenever $|X| \leqslant 12$ or $|\mathcal{F}| \leqslant 50$, from work of Vučković and Živković [8] and of Roberts and Simpson [7]. We note that Reimer [6] proved that the average size of a set in an arbitrary finite union-closed family $\mathcal{F}$ is at least $\frac{1}{2} \log _{2}(|\mathcal{F}|)$; this yields (by averaging) a good approximation to the Union-Closed 
Conjecture in the case where $\mathcal{F}$ is large, e.g. it implies that there is an element contained in at least an $\Omega(1)$-fraction of the sets in $\mathcal{F}$, in the case where $|\mathcal{F}|=2^{\Omega(n)}$.

If $X$ is a set and $\mathcal{F}$ is a family of subsets of $X$, we say $\mathcal{F}$ is transitive if the automorphism group of $\mathcal{F}$ acts transitively on $X$. (The automorphism group of $\mathcal{F}$ is the set of all permutations of $X$ that preserve $\mathcal{F}$.) Informally, $\mathcal{F}$ is transitive if all points of $X$ 'look the same' with respect to $\mathcal{F}$. Even the special case of the Union-Closed Conjecture for transitive families is wide open.

In this note, we prove the conjecture in the special case where $X$ is $\mathbb{Z}_{n}$, the cyclic group of order $n$, and $\mathcal{F}$ is the (transitive) union-closed family consisting of all unions of cyclic translates of some fixed set. This is a question asked in the Polymath project [5].

Theorem 1. Let $n \in \mathbb{N}$, and let $R \subseteq \mathbb{Z}_{n}$ with $R \neq \varnothing$. Let $\mathcal{F}=\left\{A+R: A \subseteq \mathbb{Z}_{n}\right\}$ be the set of all unions of cyclic translates of $R$. Then the average size of a set in $\mathcal{F}$ is at least $n / 2$. In particular, the Union-Closed Conjecture holds for $\mathcal{F}$.

Our proof is surprisingly short. In fact, we establish the following slightly more general result.

Theorem 2. Let $(G,+)$ be a finite Abelian group, and let $R \subseteq G$ with $R \neq \varnothing$. Let $\mathcal{F}=\{A+R: A \subseteq G\}$ be the set of all unions of translates of $R$. Then the average size of a set in $\mathcal{F}$ is at least $|G| / 2$. In particular, the Union-Closed Conjecture holds for $\mathcal{F}$.

We note that the family $\mathcal{F}$ in the statement of Theorem 2 is clearly transitive and union-closed, since $x \mapsto x+x_{0}$ is an automorphism of $\mathcal{F}$ for any $x_{0} \in G$, and $\left(A_{1}+R\right) \cup$ $\left(A_{2}+R\right)=\left(A_{1} \cup A_{2}\right)+R$ for any $A_{1}, A_{2} \subseteq G$.

We remark that it is possible to deduce a slightly weaker form of Theorem 2 from a theorem of Johnson and Vaughan (Theorem 2.10 in [3]). In fact, the result of Johnson and Vaughan, after applying a quotienting argument, yields that there is an element of $G$ contained in at least $(|\mathcal{F}|-1) / 2$ of the sets in $\mathcal{F}$. (Since $\mathcal{F}$ may have odd size, for example when $G$ is $\mathbb{Z}_{3}$ and $R=\{0,1\}$, this is not quite enough to deduce Theorem 2.) We are indebted to Zachary Chase for bringing this paper of Johnson and Vaughan to our attention.

A short explanation of our notation and terminology is in order. As usual, if $G$ is an Abelian group, and $A, B \subseteq G$, we write $A+B=\{a+b: a \in A, b \in B\}$ for the sumset of $A$ and $B$. Similarly, if $a \in G$ and $B \subseteq G$, we define $a+B=\{a+b: b \in B\}$. For any $x \in G$, we let $-x$ denote the inverse of $x$ in $G$, and for any set $A \subseteq G$, we let $-A=\{-a: a \in A\}$. We say a subset $A \subseteq G$ is symmetric if $A=-A$. If $X$ is a finite set, we write $\mathcal{P}(X)$ for the power-set of $X$.

\section{Proof of Theorem 2.}

Before proving Theorem 2, we introduce some useful concepts and notation. Let $G$ be a fixed, finite Abelian group, and let $R \subseteq G$ be fixed. For any set $A \subseteq G$, we define its $R$-neighbourhood to be

$$
N_{R}(A):=A+R
$$


and its $R$-interior to be

$$
\operatorname{Int}_{R}(A):=\{x \in G: x+R \subseteq A\} .
$$

We note that, if $R$ is symmetric and contains the identity element 0 of $G$, then the $R$ neighbourhood of any set $A$ is precisely the graph-neighbourhood of $A$ in the Cayley graph of $G$ with generating-set $R \backslash\{0\}$, and similarly, the $R$-interior of $A$ is precisely the graph-interior of $A$ with respect to this Cayley graph.

Proof of Theorem 2. Let $G$ be a fixed, finite Abelian group and let $R \subseteq G$ be a fixed, nonempty subset of $G$. Let

$$
\mathcal{F}=\{A+R: A \subseteq G\}
$$

be the union-closed family consisting of all unions of translates of $R$.

We define a function $f: \mathcal{P}(G) \rightarrow \mathcal{P}(G)$ by

$$
f(S)=-\left(G \backslash \operatorname{Int}_{R}(S)\right) \text { for all } S \subseteq G .
$$

It is clear that for any set $S \subseteq G,\left|\operatorname{Int}_{R}(S)\right| \leqslant|S|$, since for any element $r \in R$, the

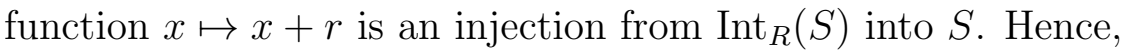

$$
|S|+|f(S)| \geqslant|G| \text { for all } S \subseteq G \text {. }
$$

Next, we observe that

$$
f(S)=(-(G \backslash S))+R \text { for all } S \subseteq G .
$$

Indeed, for any $x \in G$, it holds that $x \in f(S)$ iff $-x \notin \operatorname{Int}_{R}(S)$ iff $(-x+R) \cap(G \backslash S) \neq \varnothing$ iff $x \in(-(G \backslash S))+R$. It follows that $f(\mathcal{P}(G)) \subseteq \mathcal{F}$.

Finally, we observe that the restriction $\left.f\right|_{\mathcal{F}}$ is an injection. This might seem surprising at first glance, but it follows immediately from the fact that

$$
N_{R}\left(\operatorname{Int}_{R}(A+R)\right)=A+R \text { for all } A \subseteq G .
$$

To see (3), let $S=A+R$ and observe that $N_{R}\left(\operatorname{Int}_{R}(S)\right) \subseteq S$ holds by definition (in fact for any set $S)$. On the other hand, if $S=A+R$, then we have $A \subseteq \operatorname{Int}_{R}(S)$ and therefore $S=A+R \subseteq N_{R}\left(\operatorname{Int}_{R}(S)\right)$. Hence, $S=N_{R}\left(\operatorname{Int}_{R}(S)\right)$, as required.

Putting everything together, we see that $\left.f\right|_{\mathcal{F}}$ is a bijection from $\mathcal{F}$ to itself and satisfies

$$
|S|+|f(S)| \geqslant|G| \text { for all } S \in \mathcal{F} \text {. }
$$

Therefore,

$$
\frac{1}{|\mathcal{F}|} \sum_{S \in \mathcal{F}}|S|=\frac{1}{2|\mathcal{F}|} \sum_{S \in \mathcal{F}}(|S|+|f(S)|) \geqslant \frac{1}{2|\mathcal{F}|} \sum_{S \in \mathcal{F}}|G|=|G| / 2,
$$

proving the first part of the theorem. It follows that

$$
\frac{1}{|G|} \sum_{x \in G} \frac{|\{S \in \mathcal{F}: x \in \mathcal{F}\}|}{|\mathcal{F}|}=\frac{1}{|G|} \frac{1}{|\mathcal{F}|} \sum_{S \in \mathcal{F}}|S| \geqslant 1 / 2,
$$

so by averaging, there exists $x \in G$ such that at least half the sets in $\mathcal{F}$ contain $x$, and so the Union-Closed Conjecture holds for $\mathcal{F}$. 


\section{References}

[1] I. Balla, B. Bollobás and T. Eccles, Union-closed families of sets. J. Combin. Theory (Series A), 120 (2013), 531-544.

[2] D. Duffus, in: I. Rival (Ed.), Graphs and Order. Reidel, Dordrecht, Boston, 1985, p. 525 .

[3] R. T. Johnson and T. P. Vaughan, On Union-Closed Families, I. J. Combin. Theory (Series A), 84 (1998), 242-249.

[4] I. Karpas, Two Results on Union-Closed Families. Preprint, August 2017. arXiv: 1708.01434.

[5] Polymath11: Frankl's Union-Closed Conjecture. https://gowers . wordpress .com/ 2016/01/29/func1-strengthenings-variants-potential-counterexamples/.

[6] D. Reimer, An average set size theorem. Combin. Probab. Comput., 12 (2003), 89-93.

[7] I. Roberts and J. Simpson, A note on the union-closed sets conjecture. Australas. J. Combin., 47 (2010), 265-267.

[8] B. Vučković and M. Živković, The 12-element case of Frankl's conjecture. IPSI Transactions on Advanced Research, January 2017, Paper 9. 